\title{
基于改进共空间模式与视觉反馈的闭环脑机接口*
}

\author{
任士金金 ${ }^{1,2}$ 王卫群 ${ }^{1,2}$ 侯增广 1,2 陈霸东 ${ }^{3}$ 石伟国 ${ }^{1,2}$ 王佳星 ${ }^{1,2}$ 梁 旭 ${ }^{1,2}$ \\ (1. 中国科学院自动化研究所 北京 100190; \\ 2. 中国科学院大学 北京 100049 ;
}

3. 西安交通大学人工智能与机器人研究所 西安 710049)

\begin{abstract}
摘要: 为提高脑卒中等神经损伤患者在下肢康复训练过程中的主动参与度, 设计了基于人体下肢运动想象与视觉反馈的在线 闭环脑机接口, 并建立了基于互相关熵诱导度量与子频带分析的改进共空间模式算法, 提高人体下肢运动意图的识别率。针 对运动想象脑电信号信噪比低和难以精确识别等问题, 在传统共空间模式算法基础上, 利用互相关熵诱导度量准则改进其目 标函数, 实现了目标函数中距离项属性的动态调整, 降低对噪声的敏感性, 提高算法鲁棒性; 利用脑电信号不同频段蕴含信 息不同的特点，使用 9 个子频带滤波器对信号进行滤波，对每个子频带信号分别提取特征，并进行特征融合，建立基于互相 关熵诱导度量与子频带分析的改进共空间模式算法。其次, 基于人体下肢运动想象的脑控试验范式, 收集下肢运动想象(空想、 脚动和腿动)的脑电数据, 采用支持向量机(SVM)建立分类模型, 优化设计模型参数。在上述研究基础上, 建立了以改进共空 间模式为特征提取算法, SVM 为分类器的脑机接口。进而, 在被试执行运动想象的同时, 通过虚拟现实场景中虚拟人物的 肢体动作给予用户视觉反馈, 构建了闭环的脑机交互系统。通过试验验证了改进共空间模式算法的有效性和闭环脑机接口的 可行性, 初步实现了闭环脑机交互接口。
\end{abstract}

关键词: 运动想象; 脑机接口; 互相关熵诱导度量；共空间模式

中图分类号: TG156

\section{Closed Loop Brain Computer Interface Based on Improved Common Spatial Patterns and Visual Feedback}

\author{
REN Shixin ${ }^{1,2}$ WANG Weiqun ${ }^{1,2}$ HOU Zengguang ${ }^{1,2}$ CHEN Badong ${ }^{3}$ \\ SHI Weiguo ${ }^{1,2}$ WANG Jiaxing ${ }^{1,2}$ LIANG Xu ${ }^{1,2}$ \\ (1. Institute of Automation Chinese Academy of Sciences, Beijing 100190; \\ 2. University of Chinese Academy of Sciences, Beijing 100049; \\ 3. Institute of Artificial Intelligence and Robotics Xi'an Jiaotong University, Xi'an 710049)
}

\begin{abstract}
In order to improve the active participation of patients with stroke or nerve injury in the lower limb rehabilitation training, an on-line closed loop brain computer interface is designed based on human lower limb motor imagery and visual feedback. And an improved common spatial pattern algorithm, which based on the correntropy induced metric and sub-frequency band analysis, is established to improve the recognition rate of the motor intention. Due to the low signal noise ratio and classification accuracy of the motor imagery EEG signals, correntropy induced metric is adopted to improve the objective function of the traditional common spatial patterns (CSP). The distance term of the objective function can be adjusted dynamically to alleviate the negative effects of noise. Because the different frequency band signals have different information, nine sub-frequency bandpass filters are used to filter the signal. And the features extracted from each sub-band signal are fused. Therefore, the improved common spatial pattern algorithm based on the correntropy induced metric and sub-frequency band analysis is established. Then, based on the brain control experiment paradigm of human lower limb motor imagery, EEG data of lower limbs motor imagery (idle, foot and leg) are collected. Support vector machine (SVM) is optimized as a classification model for the motor imagery. Based on the study above, a brain computer interface based on improved common spatial pattern algorithm and SVM is built. When participant images the movements, the user's visual feedback is given to the user through the body movements of the virtual character in the virtual reality scene, and a closed loop
\end{abstract}

* 国家自然科学基金(91648208, 91848110)、北京市自然科学基金(3171001, L172050)和中国科学院战略性先导科技专项(B 类, XDB32000000)资助 项目。20181015 收到初稿, 20190124 收到修改稿 
brain computer interaction system is constructed. Experiments verified the effectiveness of the improved common space algorithm and the feasibility of closed-loop brain computer interface, and the closed loop interaction between the brain and computer is achieved initially.

Key words: motor imagery; brain-computer interface; correntropy induced metric; common spatial patterns

\section{0 前言}

研究表明, 脑卒中和脊髓损伤患者经过长时间 的康复运动训练, 可以改善其运动功能 ${ }^{[1]}$ 。康复机 器人凭借其运动重复性好, 控制精确高和可重复编 程等优势, 可以较好地实施医生制定的康复训练策 略, 代替康复治疗师进行繁重的体力劳动, 缓解目 前国内现有康复医疗资源短缺问题。

目前, 利用智能康复机器人帮助患者进行康复 训练, 已经成为国内外机器人领域研究热点 ${ }^{[2-4]}$ 。对 于改善患者运动功能的康复机器人来说, 康复策略 主要分为两种: 一种是被动康复训练, 针对患病初 期运动功能暂时丧失, 以及病情严重导致运动能力 残存较少的病人。由康复机器人带动患者进行被动 训练, 防止其肌肉萎缩。但在这个过程中, 患者主 动参与度非常低, 完全是进行被动运动, 无法有效 调动患者积极性, 功能恢复较为缓慢; 另一种就是 主动康复训练, 运动训练的发起者是患者, 机器人 通过检测和分析患者的运动意图信息, 将其运动意 图转化为机器人控制命令, 协助患者完成其训练动 作, 提高患者主动参与度。

判断运动意图的方式主要是基于力位信息和基 于生理信号的检测和分析。如意大利的 IQBAL 等 ${ }^{[5]}$ 采用人与外骨骼之间交互力反馈, 来识别人体运动 意图; 北京航空航天大学 LI 等 ${ }^{[6]}$ 检测手指的力矩驱 动人体手指康复机器人。中国科学院自动化研究 所张峰等 ${ }^{[7]}$ 通过分析下肢表面肌电信号 (Surface electromyography signal, sEMG)中的意图信息, 实现 患者与康复机器人交互, 让机器人协助患者进行主 动训练。上述两种信号, 无论是力信号还是 $\mathrm{sEMG}$ 信号, 都是属于人体远端肢体信号, 存在延时问题; 而且对于肌力较弱的患者来说, 难以从肢体信号中 提取有效信息。

人体脑皮层的信号可以先于远端肢体的信息反 映出患者运动意图, 基于脑机接口(Brain computer interface, BCI)的智能康复机器人对患者的神经系统 康复有着重要的作用 ${ }^{[8]}$ 。杜克大学研究表明, 结 合 BCI、康复机器人和触觉反馈的长期康复训练 可以帮助完全脊髓损伤患者的部分神经再生 ${ }^{[9]}$ 。

GOMEZ-RODRIGUEZ 等 ${ }^{[10]}$ 采用 Barret 七自由度的
机械臂, 让患者通过脑机接口控制其运动, 进而刺 激脑皮质层的运动功能区。

作为脑机接口信息源头的脑电信号包括多种形 式, 其中有 P300 相关事件电位(在一个刺激发生后 的 $300 \mathrm{~ms}$ 附近有一个正电位增强), 稳态视觉诱发 电位(固定频率的视觉刺激后大脑皮层产生的响 应), 以及运动想象信号(Motor imagery, MI), 基于 前两种模式的脑机接口研究较多, 在残疾人脑控外 设应用广泛, 如脑控手机拨号和打字, 脑控轮椅 ${ }^{[11]}$ 。 但是这两种模式都是基于外部刺激后，大脑皮层的 相关响应, 是一种非自主控制行为。在脑控过程中, 大脑只起到了接收刺激然后输出响应的作用, 脱离 刺激后, 被试者自己无法单独产生, 这对于需要康 复的患者来说是不适用的。运动想象信号则是通过 人体自身想象运动后, 在相关脑皮层检测到的一种 电信号, 完全由人体自发产生。由其主动意识产生 神经系统的活动, 强调了患者的作用, 因此在基于 脑机接口的康复机器人系统中采用运动想象信号作 为控制命令来源, 将有利于调动患者参与积极性 ${ }^{[2]}$ 。

如何从运动想象的脑电信号中快速和高效地提 取出意图信息一直以来都是一个难点问题。一方面是 因为脑电信号本身存在低的信噪比, 容易受干扰, 特 征提取困难, 分类准确率较低; 另一方面是难以充分 调动被试者参与到运动想象过程中, 被试者在运动想 象过程中易走神。另外, 由于脑皮层功能区负责上肢 活动的区域较大, 目前运动想象动作大部分局限于上 肢活动, 因下肢活动区域小，难度大，基于下肢的脑 机接口并不多见。在本文中, 通过设计试验范式和采 用改进算法能有效地区分下肢运动想象信号。

针对脑电信号特征提取问题, 共空间模式 (Common spatial patterns, CSP)特征提取方法被广泛 采用, 通过建立空间滤波器, 将两类信号的方差最 大化, 然后提取滤波后的特征进行分类。但因脑电 信号易受噪声影响, CSP 在实际使用过程中, 尤其 是在线实时分类时, 效果不稳定, 对异常数据敏感。 为降低噪声对特征提取的影响, 基于互相关熵诱导 度量准则(Correntropy induced metric, CIM)改进了 CSP 的目标函数, 降低对异常数据和噪声的敏感性, 提高算法鲁棒性; 同时对信号进行子频带 (Sub frequency band, SF)滤波, 对滤波后的 9 个频带信号 分别提取特征, 然后采用支持向量机(Support vector 
machine, SVM)进行分类, 结果表明基于改进算法提 取的特征分类准确率得到有效提升。同时, 以改进 算法为特征提取算法, SVM 为分类器的脑机接口被 建立。进而将脑机接口与基于 Unity 开发脑控虚拟 场景相结合, 被试可以通过想象腿部和脚部运动来 控制虚拟情景中的人物动作, 并给予用户视觉反馈, 形成闭环交互。经过试验验证后, 初步实现了闭环 脑机交互接口。

\section{1 脑机接口系统介绍}

\section{1 数据采集}

被试者佩戴 40 通道的脑电电极帽, 通过 NeuroScan 公司的 Grael V2 EEG 采集系统进行脑电 信号的采集。由于运动想象主要涉及的是脑皮层的 运动功能区, 因此仅对文献中常采用的运动功能区 附近的 4 个通道数据做了记录，其分别是 “ $\mathrm{C} 3$ ”, “CZ”，“C4” 和 “FZ”。为了保证脑电信号质量, 在对 4 个通道注射导电亳后, 当每个电极的阻抗值 都稳定在 $5 \mathrm{k} \Omega$ 以下才开始进行信号记录, 脑电信号 采集频率设置为 $256 \mathrm{~Hz}$ 。

\section{2 虚拟交互场景}

基于 Unity 平台开发了虚拟人物场景(图 1), 场景 中人物可以更好地帮助被试者关注想象的动作，同时 人物的下肢动作是可以由被试者控制的。来自被试的 脑电信号经过分类器后，转变为人物肢体控制命令; 同时, 在场景中给被试设置积分环节, 当成功达到系 统提示操作后, 会以分数的形式给被试者加以反馈, 从而在被试者和场景之间形成一个闭环交互回路。具 体的交互方式将会在试验部分详细介绍。

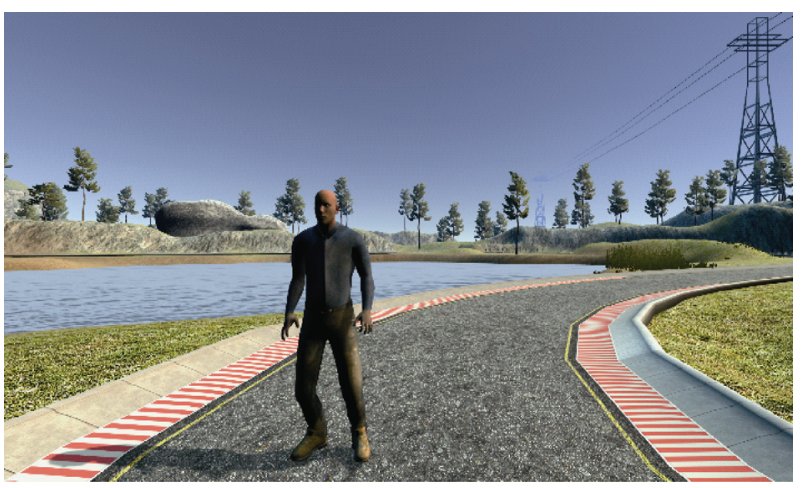

图 1 脑控虚拟人物

2 共空间模式算法及其改进算法

\section{1 传统共空间模式方法}

共空间模式是一种在脑电信号特征提取时被广
泛采用的一种方法, 特别是在基于运动想象的脑机 接口中 ${ }^{[13]}$ 。共空间模式主要针对二分类问题，目的 是计算出一个空间滤波器，能将两类中一类的方差 最大化，同时保证另一类的方差最小。然后提取差 异最大化后的特征, 进而降低分类难度。以脑电信 号为例, $S_{1}$ 和 $S_{2}$ 分别代表一类信号, 维度是 $M \times N$, 其中 $N$ 代表信号样本时间点数， $M$ 代表通道数。每 类协方差矩阵计算如下

$$
\boldsymbol{X}_{i}=\frac{\boldsymbol{S}_{i} \boldsymbol{S}_{i}^{\mathrm{T}}}{\operatorname{tr}\left(\boldsymbol{S}_{i} \boldsymbol{S}_{i}^{\mathrm{T}}\right)}
$$

式中, $i$ 代表第 $i$ 类, $\operatorname{tr}$ 表示求矩阵的迹, $\mathrm{T}$ 表示矩 阵的转置。如果每类信号有多组, 则将多组的协方 差矩阵求平均 $\bar{X}_{i}$, 在求平均之前, 多组信号必须经 过同样的滤波，去均值和缩放操作。在得到协方差 矩阵后, CSP 的目标就是寻找能将两类信号方差最 大化的空间滤波器, 其可以通过最大化以下目标函 数得到

$$
J(\boldsymbol{\omega})=\frac{\boldsymbol{\omega}^{\mathrm{T}} \overline{\boldsymbol{X}}_{1} \boldsymbol{\omega}}{\boldsymbol{\omega}^{\mathrm{T}} \overline{\boldsymbol{X}}_{2} \boldsymbol{\omega}}
$$

式中, $\omega$ 是空间滤波器, 将目标函数重写如下

$$
J(\boldsymbol{\omega})=C \frac{\left\|\boldsymbol{\omega}^{\mathrm{T}} \boldsymbol{S}_{1}\right\|_{2}^{2}}{\left\|\boldsymbol{\omega}^{\mathrm{T}} \boldsymbol{S}_{2}\right\|_{2}^{2}}
$$

式中, $C$ 表示常数, 可以看出 CSP 的目标函数是 $L_{2}$ 范数形式, 因此 CSP 是对噪声比较敏感的, 当信号 受到干扰时, $L_{2}$ 范数形式将会使异常数据影响增 大，这对于分类是十分不利的。为了改变对异常数 据敏感的特点, 在 CSP 算法基础上进行改进, 提出 了一种更为鲁棒的算法一基于互相关熵诱导度量与 子频带分析的共空间模式算法。

\section{2 基于互相关熵诱导度量与子频带分析的共空 间模式算法(SFCSP-CIM)}

由于 $L_{2}$ 范数形式的目标函数限制了 CSP 的算 法效果，导致异常值对整体影响较大。因此，采用

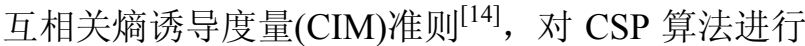
改进, 改善目标函数对异常值敏感的问题。CIM 是 基于再生希尔伯特空间 ${ }^{[15]}$ 中的互相关熵诱导度量 准则，其距离度量方式能够在不同的动态区域分别 表现出 $L_{2}, L_{1}$ 和 $L_{0}$ 范数性质 ${ }^{[14]}$, 对噪声的变化能 够进行动态响应, 有针对性地降低噪声带来的影响, 提升算法的鲁棒性。

下面是对基于 CIM 准则对传统 CSP 方法的改 进介绍, 假设给定两个变量 $\boldsymbol{a}=\left[a_{1}, a_{2}, \cdots, a_{3}\right]$ 和 $\boldsymbol{b}=\left[b_{1}, b_{2}, \cdots, b_{3}\right]$, 它们的熵计算定义如下 


$$
V(\boldsymbol{a}, \boldsymbol{b})=\frac{1}{N} \sum_{i=1}^{N} k\left(a_{i}, b_{i}\right)
$$

式中, $k(\cdot, \cdot)$ 采用的是高斯核

$$
k(a, b)=k(a-b)=\frac{1}{\sqrt{2 \pi} \sigma} \exp \left(-\frac{\|a-b\|^{2}}{2 \sigma^{2}}\right)
$$

式中, $\sigma$ 是高斯核宽度。此核函数可以将变量 $\boldsymbol{a}$ 和 $\boldsymbol{b}$ 变换到再生希尔伯特空间 ${ }^{[11]}$ 。在此空间里, 两个变 量之间的 “欧式距离” 被定义为

$$
\left[(\hat{\boldsymbol{a}}-\hat{\boldsymbol{b}})^{T}(\hat{\boldsymbol{a}}-\hat{\boldsymbol{b}})\right]^{\frac{1}{2}}=\sqrt{2 N}[k(0)-V(\boldsymbol{a}, \hat{\boldsymbol{b}})]
$$

式中, $\hat{\boldsymbol{a}}$ 和 $\hat{\boldsymbol{b}}$ 是经过变换后再生希尔伯特空间里的 变量, 在式(6)的基础上经过变换, 将变换后的式子 称为 CIM, 计算如下

$$
\operatorname{CIM}(\boldsymbol{a}, \boldsymbol{b})=[k(0)-V(\boldsymbol{a}, \boldsymbol{b})]^{\frac{1}{2}}
$$

在这里将 CIM 作为两变量之间距离的一种度 量准则。文献[14]表明, CIM 会随着变量 $\boldsymbol{a}$ 和 $\boldsymbol{b}$ 之间 的距离从近到远，依次展现 $L_{2}, L_{1}$ 和 $L_{0}$ 范数性质, 可用于减小异常数据的干扰。因此, 将 CIM 准则和 传统 CSP 的目标函数相结合, 改进其成如下形式

$$
\tilde{J}(\boldsymbol{\omega})=\frac{\operatorname{CIM}\left(\boldsymbol{\omega}^{\mathrm{T}} \boldsymbol{S}_{1}, 0\right)}{\operatorname{CIM}\left(\boldsymbol{\omega}^{\mathrm{T}} \boldsymbol{S}_{2}, 0\right)}=\frac{\left[k(0)-V\left(\boldsymbol{\omega}^{\mathrm{T}} \boldsymbol{S}_{1}, 0\right)\right]^{\frac{1}{2}}}{\left[k(0)-V\left(\boldsymbol{\omega}^{\mathrm{T}} \boldsymbol{S}_{2}, 0\right)\right]^{\frac{1}{2}}}
$$

通过对 $\tilde{J}(\omega)$ 求导, 采用梯度更新的方式求取空 间滤波器 $\omega$, 使更新后的目标函数 $\tilde{J}(\omega)$ 最大化, 同
理通过最小化 $\tilde{J}(\omega)$, 也可以得到空间滤波器 $\omega^{\prime}$, 详细的求解过程, 可以参考文献[16]。脑电信号 $S_{1}$ 和 $S_{2}$ 有 $M$ 个通道, 那么最多可以分别获得 $k$ 个空间滤 波器 $\boldsymbol{\omega}$ 和 $\boldsymbol{\omega}^{\prime}(1 \leqslant k \leqslant M)$ 。

利用得到的空间滤波器可以对样本进行特征提 取。若有一段包含 $t$ 个离散采样点的脑电信号, 则 这段样本的特征可以通过以下方式来计算获得

$$
f=\left(\frac{1}{t} \sum_{i=1}^{t}\left(1-\exp \left(-\frac{\left(\boldsymbol{\omega}^{\mathrm{T}} \boldsymbol{S}^{i}\right)^{2}}{2 \sigma^{2}}\right)\right)\right)^{\frac{1}{2}}
$$

若采用 $2 k$ 个空间滤波器 $\boldsymbol{\omega}$ 和 $\boldsymbol{\omega}^{\prime}$ 进行特征提取 的情况下，这段脑电信号特征矢量可以表示为 $\boldsymbol{f}=\left[f_{1}, \cdots, f_{k}, f_{1}^{\prime}, \cdots, f_{k}{ }^{\prime}\right]$ 。对于其他信号样本都可以 通过此公式提取特征, 然后用利用分类器进行分类。 CSP-CIM 算法可以有效降低异常点影响, 其性能已 经经过测试数据验证 ${ }^{[16]}$ 。

脑电信号所包含的频率带是比较宽的, 但对运 动想象的脑电信号分析时，往往只考虑 $4 \sim 30 \mathrm{~Hz}$ 的频率段 ${ }^{[17]}$ 。研究表明, 通过对此频率段进行细化 分频可以提取更多有用信息 ${ }^{[18]}$ 。针对每个频率带分 别提取特征可以提高信号可分性, 因此在处理数据 时, 采用先让脑电信号经过多个带通滤波器方式, 产生多个子频率带的脑电信号, 然后利用 CSP-CIM 对各个子频带信号进行特征提取，将提取的多个特 征组成新的特征矢量, 作为该段脑电信号的样本特 征，图 2 是特征提取的流程。

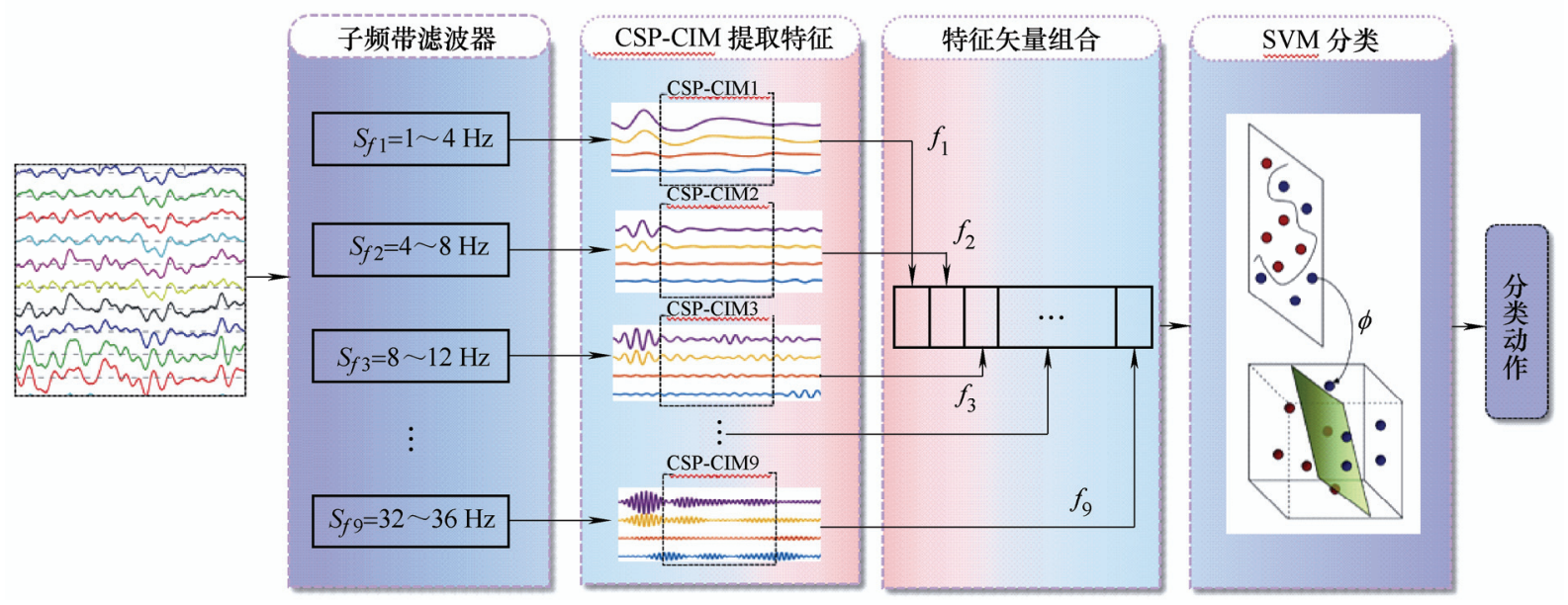

图 2 SFCSP-CIM 特征提取分类流程

如图 2 所示, 在本文中, 选取了 $1 \sim 36 \mathrm{~Hz}$ 的信 号频率带, 采用 9 个带通滤波器, 将信号滤波成 9 个频率带 $\left(S_{f 1}, \cdots, S_{f 9}\right)$, 然后分别采用 CSP-CIM 提取 每个频率带的特征组成特征矢量 $\left[f_{1}, \cdots, f_{9}\right]$, 将此特 征矢量作为该样本特征, 采用支持向量机(SVM)用 作分类器模型, 对样本进行训练分类。

\section{3 试验设计及算法验证}

本次试验分为两个阶段, 第一个阶段是采集被 试的训练数据, 进行脑电信号预处理、特征提取和 分类器建模, 然后将建立好的分类器模型提供给第 
二阶段。第二阶段就是在线脑控试验, 被试通过 系统提示, 参与到虚拟场景中, 程序实时检测被 试的脑电信号, 经过预处理和特征提取后, 由分 类器后产生控制命令, 进而达到脑控人物的目的, 同时系统也会将检测结果实时反馈给被试, 形成 闭环交互。

\section{1 离线数据采集和分类器训练}

运动想象选取的状态有三个: 第一个是想象腿 动; 第二个是想象脚动; 第三个是空想状态(idel)。 根据与脑控虚拟场景的交互模式, 主要是区分空想
和脚动状态, 以及空想和腿动状态, 因此会建立两 种分类模型。

试验采用 Eprime 软件 ${ }^{[19]}$ 设计了试验流程程序, 如图 3 所示。每次试验一共有 10 个组, 每组包含 3 个状态想象时间段，每个时间段持续 $10 \mathrm{~s}$ 。在试验 开始之前, 屏幕会显示一个 “十” 字形, 帮助被试 集中精力, 为接下来试验做好准备。每次状态想象 之前, 屏幕会给出要想象的状态提示, 同时 Eprime 会给记录的脑电数据放大器发送一个标签数据, 标 记接下来要记录的数据。

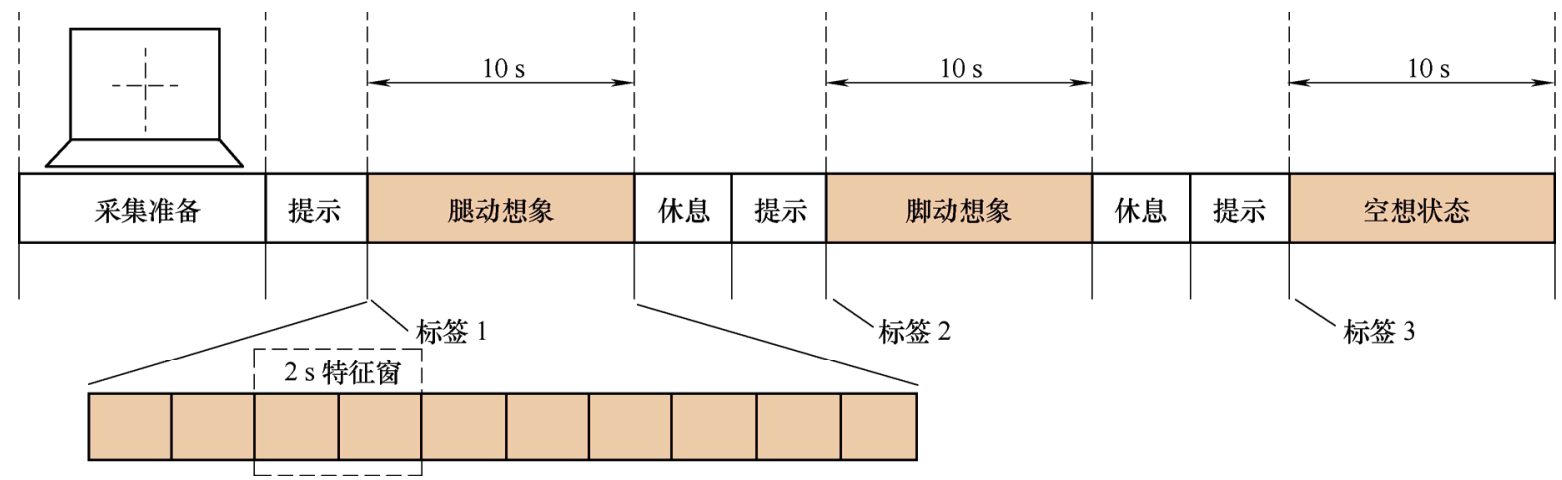

图 3 训练数据采集实验流程

在脑电信号记录完成后, 使用 Matlab 对数据进 行处理。首先, 将信号进行去均值处理, 也就是将 其去中心化。然后对信号进行子频带滤波, 如图 2 所示, 将信号滤波为 9 个频率带的信号, 采用的滤 波器是 5 阶巴特沃思滤波器。最后是对每个频率带 的信号提取特征, 在此之前, 有几个参数需要确定, 其中一个是提取特征的窗口大小。根据特征提取公 式(9), 要设置包含 $t$ 个采样点的脑电信号窗口, 在 以分类准确率最高为目标的前提下, 尝试了多种窗 口大小设置, 最终设置包含 512 个采样点的窗口, 信号采样率是 $256 \mathrm{~Hz}$, 也就是每个窗口包含 $2 \mathrm{~s}$ 的 脑电信号(图 3 特征窗), 这与文献[20]采用的窗口一 致。还有两个参数是 SFCSP-CIM 中高斯核函数宽 度 $\sigma$ 和求解滤波器 $\boldsymbol{\omega}$ 时的迭代学习率 $\eta$, 同样在经 过参数遍历寻优后, 两个分类器各自的 $\sigma$ 和 $\eta$ 最优 值分别确定为 $[0.1,0.1]$ 和 $[0.7,0.1]$ 。分类器采用的是

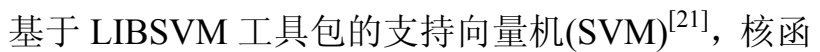
数为径向基函数 (RBF)。为空想和脚动建立的分类器 为 SVM1, 空想和腿动建立的分类器为 SVM 2 。在 分类器建模过程中, 训练数据和测试数据来自多次 试验记录, 并采用交叉验证结果作为寻优准则。

在上述操作完成后, 优化的分类器模型 SVM1 和 SVM2 被分别建立; 同时, 还获取了每个模型所 在组的空间滤波器组 $\boldsymbol{\omega}$, 每个频带都选取了两个滤 波器 $\omega_{i}=\left[\omega_{i}, \omega_{i}{ }^{\prime}\right](1 \leqslant i \leqslant 9)$, 因此, 每个分类器模型
都对应滤波器组 $\omega=\left(\left[\omega_{1}, \omega_{1}{ }^{\prime}\right], \cdots,\left[\omega_{9}, \omega_{9}{ }^{\prime}\right]\right)$ 。对于未 知的脑电信号, 在经过子频带滤波后, 可以直接利 用这些空间滤波器进行特征提取, 然后由分类器模 型给出分类结果。

\section{2 在线脑控试验}

当被试选择开始后，分类程序可以根据检测到 的想象状态转化为命令, 在线控制场景中人物。具 体步骤如下。

(1) 被试点击开始后, 系统会发出声音提示, 只要被试在提示后保持想象腿动状态, 在此过程中 一旦程序检测到腿动状态, 场景中人物双腿就会开 始运动, 然后程序进入下一步。

(2) 在人物双腿运动后, 被试可以进入短暂休 息状态, 然后跟随系统提示操作。在 $20 \mathrm{~s}$ 后, 系统 会进入积分阶段，积分阶段只有 $10 \mathrm{~s}$ 时间，系统会 让用户保持想象腿部运动状态，如果用户在 $10 \mathrm{~s}$ 内 成功达到 3 次，那么积分 $A$ 会增加 1 分; 如果没有 达到, 则不计分。操作完成进入下一步。

(3) 在上次操作完成间隔 $20 \mathrm{~s}$ 后, 系统会提示 被试进入运动维持阶段, 本阶段同样只有 $10 \mathrm{~s}$ 时间, 如果用户选择继续, 被试必须在 $10 \mathrm{~s}$ 内保持想象脚 动状态, 每当检测到脚动, 人物会有一个脚动反馈, 同时, $10 \mathrm{~s}$ 内成功达到 3 次, 积分 $B$ 增加 1 分, 并 继续进行步骤(2); 如果保持空想状态, 达到 3 次后, 会结束本次试验。 
每次试验结束都会后, 积分数据 $A$ 和 $B$ 都会反 馈给用户, 积分 $A$ 反应想象腿动的成功次数, $B$ 想 象脚动的成功次数, 被试想同时要得到高分, 必须 在过程中尽可能多地想象腿动和脚动状态。同时, 积分对用户来说, 也起到了激励和正反馈作用, 调 动了被试参与的积极性。如图 4 所示, 被试进行在 线脑控试验。

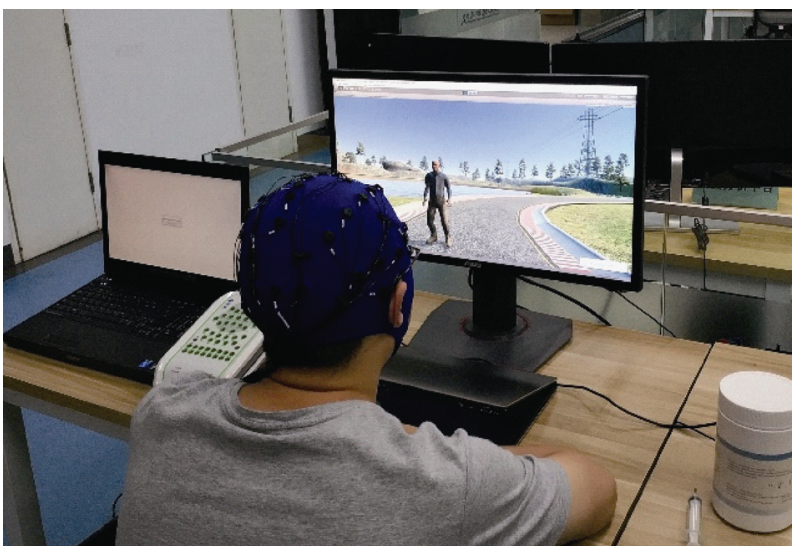

图 4 在线脑控试验

\section{4 试验结果}

\section{1 离线数据训练结果}

训练数据一共采集了 60 个时间段的数据, 用来 训练分类器, 交叉验证的结果用来评价分类器性能。 为了更客观的比较, 对 CSP 也采用了子频带过滤, 在这称为 SFCSP, 其分类结果用作对比。利用离线 数据共对比了基于 CSP、CSP-CIM、SFCSP 和 SFCSP-CIM 四种方法的分类正确率(ACR), 此处负 样本指的是腿动和脚动状态, 空想状态是正样本。 前两种方法针对的特征样本是只经过 $1 \sim 36 \mathrm{~Hz}$ 带 通滤波器过滤后提取信号特征, 后两种是经过子频 带滤波器滤波后的提取信号特征。结果如表 1 所示, 其中 SVM1 表示空想与脚动分类模型, SVM2 表示 空想与腿动分类模型。

表 1 离线数据建模的分类器性能

\begin{tabular}{ccccc}
\hline \multirow{2}{*}{ 编号 } & \multicolumn{4}{c}{ 分类准确率(\%) } \\
\cline { 2 - 5 } & CSP & CSP-CIM & SFCSP & SFCSP-CIM \\
\hline SVM1(ACR) & 73 & 65 & 68 & 82 \\
SVM2(ACR) & 65 & 70 & 67 & 74 \\
\hline
\end{tabular}

从表 1 可以看出, SFCSP-CIM 算法的准确率都 是最高的。通过增加信号子频带分析后, 对于 CSP-CIM 准确率提升非常明显, 平均提升约 16\%; 对 CSP 来说, 只有一类分类准确率有提升, 另一 类则下降了, 这可能是由 CSP 算法的鲁棒性较弱 造成的。

\section{2 在线脑控结果}

根据第 2.2 节中步骤所示, 被试进行试验时, 如果被试一直积极参与, 不自动选择退出, 且分类 器正确分类情况下, 可以在步骤(2)、(3)之间多次循 环。每次循环可以由被试选择终止或者分类器误判 意外终止, 所以为了方便统计结果, 只关注用户在 步骤(2)、(3)的完成情况, 这两步都是以 $10 \mathrm{~s}$ 为一个 单位, 前面确定的最优分类窗口时间长度是 $2 \mathrm{~s}$, 分 类器在 $10 \mathrm{~s}$ 内可以完成 5 次分类。

为了测试在线分类性能，不仅让被试根据系统 提示完成腿动和脚动的运动想象，而且还在提示中 测试了空想分类准确率。如图 5 所示, 图 5a、5b 是空想和脚动在线分类结果, “ 1 ” 是空想的标签, “ -1 ” 是脚动标签, 图中每个点是 $2 \mathrm{~s}$ 窗的实时分 类结果，5 个窗口结果融合后给出一个综合分类结 果, 以供积分使用。从图 5c、5d 中可以看出, 对于 空想( “ 1 ”)和脚动( “ -1 ”) 两种状态, $2 \mathrm{~s}$ 窗口的在 线分类准确率约为 $79 \%$, 在 5 个窗口综合后, 空想 10 次有 1 次错误，而脚动全部正确; 同样对于空想 与腿动状态， $2 \mathrm{~s}$ 窗口的实时分类准确率约为 $72 \%$, 但是 5 个窗口综合后的结果显示，空想仅有 1 次错 误, 腿动全部正确。

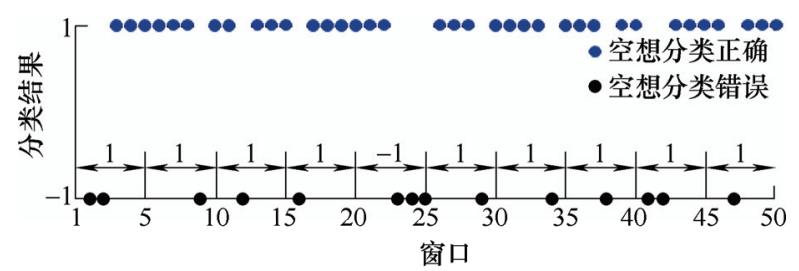

(a)

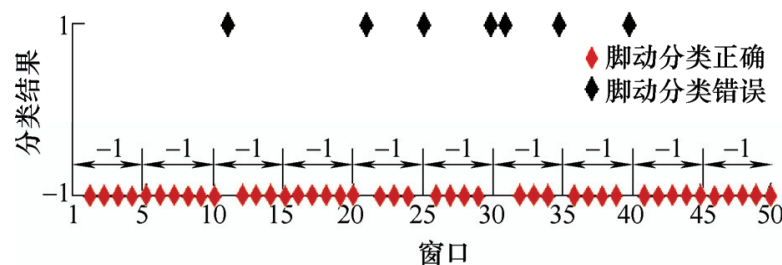

(b)

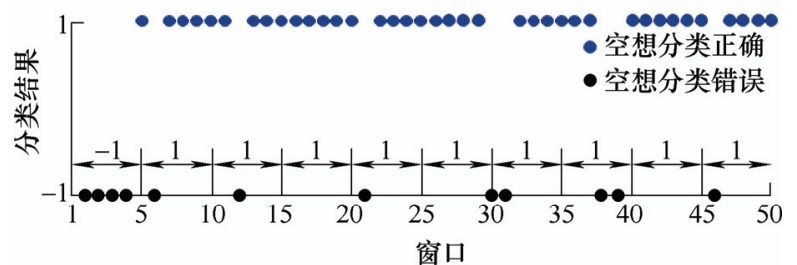

(c)

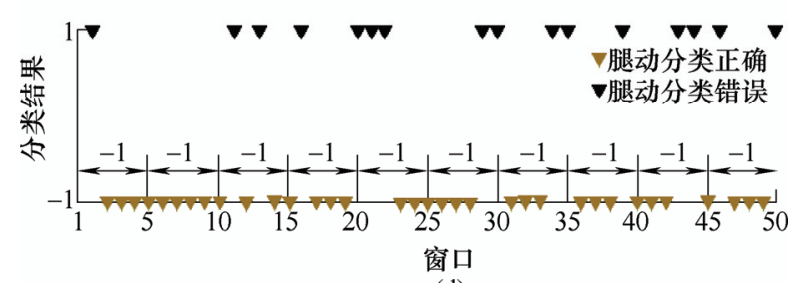

(d)

图 5 在线分类结果 


\section{5 结论}

（1）针对脑电信号信噪比低, 易受干扰, 传统 CSP 对噪声敏感的问题, 建立了基于互相关熵诱导 度量和子频带分析的改进共空间模式算法, 可有效 提取运动想象脑电信号特征, 试验对比结果表明该 方法可有效提高了分类准确率。

（2）设计了基于运动想象的试验范式，选定了 目前研究较少的人体下肢运动想象作为研究对象, 经过离线数据采集, 特征提取和分类器优化设计后 建立了脑机接口。

(3) 通过在线试验, 验证了本文改进算法的有 效性和闭环脑机接口的可行性, 通过虚拟现实场景 中虚拟人物的肢体动作给予用户视觉反馈, 初步实 现了闭环脑机交互接口。

未来将结合本文的闭环脑机接口技术和课题组 研发的下肢康复机器人为患者制定实用有效的康复 策略。

\section{参 考 文 献}

[1] WOLPAW J R, TENNISSEN A M. Activity-dependent spinal cord plasticity in health and disease[J]. Annual Review of Neuroscience, 2001，24(1): 807-843.

[2] 侯增广, 赵新刚, 程龙, 等. 康复机器人与智能辅助系 统的研究进展 [J]. 自动化学报, 2016, 42(12): 1765-1779.

HOU Zengguang, ZHAO Xingang, CHENG Long, et al. Recent advances in rehabilitation robots and intelligent assistance systems[J]. Acta Automatica Sinica, 2016, 42(12): 1765-1779.

[3] WANG Congzhe, FANG Yuefa, GUO Sheng. Multi-objective optimization of a parallel ankle rehabilitation robot using modified differential evolution algorithm[J]. Chinese Journal of Mechanical Engineering, 2015, 28(4): 702-715.

[4] 倪自强, 王田苗, 刘达. 医疗机器人技术发展综述 [J]. 机械工程学报, 2015，51(13): 45-52.

Ni Ziqiang, WANG Tianmiao, LIU Da. Survey on medical robotics[J]. Journal of Mechanical Engineering, 2015, 51(13): 45-52.

[5] IQBAL J, TSAGARAKIS N G, CALDWELL D G. Human hand compatible underactuated exoskeleton robotic system[J]. Electronics Letters, 2014, 50(7): 494-496.

[6] LI Jiting, WANG Shuang, WANG Ju, et al. Development of a hand exoskeleton system for index finger rehabilitation[J]. Chinese Journal of Mechanical
Engineering, 2012, 25(2): 223-233.

[7] ZHANG Feng, LI Pengfeng, HOU Zenggou, et al. sEMG-based continuous estimation of joint angles of human legs by using BP neural network[J]. Neurocomputing, 2012, 78(1): 139-148.

[8] SOEKADAR S R, BIRBAUMER N, SLUTZKY M W, et al. Brain machine interfaces in neurorehabilitation of stroke[J]. Neurobiology of disease, 2015，83: 172-179

[9] DONATI A R C, SHOKUR S, MORYA E, et al. Long-term training with a brain-machine interface-based gait protocol induces partial neurological recovery in paraplegic patients[J]. Scientific Reports, 2016, 6: 30383.

[10] GOMEZ-RODRIGUEZ M, GROSSE-WENTRUP M,

HILL J, et al. Towards brain-robot interfaces in stroke rehabilitation-[C]// IEEE International Conference on Rehabilitation Robo-tics, June 29 - July 1, 2011, Zurich, Switzerland, Piscataway: IEEE, 2011: 1-6.

[11] LI Yuanqing, PAN Jiahui, WANG Fei, et al. A hybrid BCI system combining P300 and SSVEP and its application to wheelchair control[J]. IEEE Transactions on Biomedical Engineering, 2013, 60(11): 3156-3166.

[12] 唐智川, 孙守迁, 张克俊. 基于运动想象脑电信号分类 的上肢康复外骨骼控制方法研究 $[\mathrm{J}]$. 机械工程学报, 2017, 53(10): 60-69.

TANG Zhichuang, SUN Shouqian, ZHANG Kejun. Research on the control method of an upper-limb rehabilitation exoskeleton based on classification of motor imagery EEG[J]. Journal of Mechanical Engineering, 2017, 53(10): 60-69.

[13] NASIHATKON B, BOOSTANI R, JAHROMI M Z. Aneff-icient hybrid linear and kernel CSP approach for EEG feature extraction[J]. Neurocomputing, 2009, 73(1-3): 432-437.

[14] LIU W, POKHAREL P P, PRINCIPE J C. Correntropy: Properties and applications in non-Gaussian signal process-ing[J]. IEEE Transactions on Signal Processing, 2007, 55(11): 5286-5298

[15] ARONSZAJN N. Theory of reproducing kernels[J]. Transactions of the American Mathematical Society, 1950, 68(3): 337-404.

[16] DONG J, CHEN B, LU N, et al. Correntropy induced metric based common spatial patterns[C]// IEEE 27th International Workshop on. IEEE Machine Learning for Signal Processing, September 25-28, 2017, Tokyo, Japan, Piscataway: IEEE, 2017: 1-6.

[17] BAARS B J, GAGE N M. Cognition, brain, and consciousness: Introduction to cognitive neuroscience[M]. Academic Press, 2010.

[18] SUN Gufei, HU Jinglu, WU Gengfeng. A novel frequency 
band selection method for common spatial pattern in motor imagery based brain computer interface[C]// IEEE International Joint Conference on Neural Networks, July 18-23, 2010, Barcelona, Spain, Piscataway: IEEE, 2010: $1-6$.

[19] SCHNEIDER W, ESCHMAN A, ZUCCOLOTTO A. E-Prime[M]. Psychology Software Incorporated, 2002.

[20] LOTTE F, GUAN C. Regularizing common spatial patterns to improve BCI designs: Unified theory and new algorithms[J]. IEEE Transactions on biomedical Engineering, 2011, 58(2): 355-362.

[21] CHANG C C, LIN C J. LIBSVM: A library for support vector machines[J]. ACM Transactions on Intelligent Systems and Technology (TIST), 2011, 2(3): 1-27.
作者简介: 任士䧻, 男, 1993 年出生, 博士研究生。主要研究方向为康 复机器人交互控制, 脑机接口。

E-mail: renshixin2015@ia.ac.cn

王卫群(通信作者), 男, 1979 年出生, 博士, 副研究员, 硕士研究生导 师。主要研究方向为康复机器人, 人机交互控制, 生理电信号处理。 E-mail: weiqun.wang@ia.ac.cn

侯增广, 男, 1969 年出生, 博士, 研究员, 博士研究生导师。主要研究 方向为机器人与智能系统, 康复机器人与微创介入手术机器人。

E-mail: zengguang.hou@ia.ac.cn

陈霸东, 男, 1974 年出生, 博士, 教授, 博士研究生导师。主要研究方 向为信息论学习，信号处理及脑机接口。

E-mail: chenbd@mail.xjtu.edu.cn

石伟国, 男, 1994 年出生, 硕士研究生。主要研究方向为康复机器人。 E-mail: shiweiguo2017@ia.ac.cn

王佳星, 女, 1992 年出生, 博士研究生。主要研究方向为康复机器人。 E-mail: wangjiaxing2016@ia.ac.cn

梁旭, 男, 1991 年出生, 博士研究生。主要研究方向为康复机器人。

E-mail: liangxu2013@ia.ac.cn 